\title{
The Timepix3 Telescope for LHCb Upgrade RD measurements
}

\author{
Daniel Saunders* ${ }^{* \dagger}$ \\ University of Bristol \\ E-mail: dan. saunders@bristol.ac.uk
}

The Timepix 3 telescope is a high rate, data driven beam telescope created for LHCb upgrade studies, such as sensor performance for prototypes of the vertex locator (VELO) upgrade. When testing VELO prototypes the readout is identical to the telescope, and additionally, a simple way to integrate triggers from other detectors is also provided, allowing tracks to be synchronised offline with other devices under test. Examples of LHCb upgrade detectors which have been qualified with the Timepix3 telescope are the Upstream Tracker (UT), Scintillating Fibres (SciFi), Ring Imaging CHerenkov (RICH), and Time Of internally Reflected CHerenkov light (TORCH). The telescope was installed in the SPS North hall at CERN. It consists of 8 planes with $300 \mu \mathrm{m}$ p-on-n silicon sensors read out by Timepix 3 ASICs. Tracks measured with the telescope have excellent temporal ( $\sim 1 \mathrm{~ns})$ and spatial resolution ( $2 \mu \mathrm{m})$. The telescope has been operated with a rate of tracks written to disk up to $5 \mathrm{MHz}$ - limited only by conditions at the SPS. A description of the telescope and main results of the telescope performance such as track reconstruction efficiency are presented.

38th International Conference on High Energy Physics 3-10 August 2016

Chicago, USA

* Speaker.

${ }^{\dagger}$ on behalf of the LHCb Collaboration 


\section{Introduction}

$\mathrm{LHCb}$ is a flavour physics experiment based at the LHC, which searches for new physics through precise measurements of $\mathrm{CP}$ violation and the rare decays of heavy flavour measons [1]. The luminosity at LHCb is currently $4 \times 10^{32} \mathrm{~cm}^{-2} \mathrm{~s}^{-1}$, which corresponds to 1.5 interactions per bunch crossing on average. The experiment records data at a rate of $\sim 1 \mathrm{MHz}$, and is currently limited by the hardware electronics trigger. During long shut down 2 of the LHC, the luminosity at $\mathrm{LHCb}$ will be increased by a factor $\sim 5$, and the current trigger systems replaced with a new software trigger. The entire detector will be read-out at a rate of $40 \mathrm{MHz}$, and the increased rate of data taking will provide greater sensitivity for many physics analyses [4]. This requires a refit of the current $\mathrm{LHCb}$ front end readout, as well as upgrades of various sub-detectors to withstand the increase in luminosity.

The Timepix3 Telescope has been constructed to perform characterisation studies of many $\mathrm{LHCb}$ upgrade technologies using testbeams typically at CERN. The testbeam environment is one of the best available to perform these studies, given the close similarity to that planned for the upgraded detector. As well as studies for the upgrades of several LHCb subdetectors, the Timepix3 Telescope has been used extensively for sensor studies of the upgraded VELO - a hybrid pixel detector, whose sensors can also be bump bonded

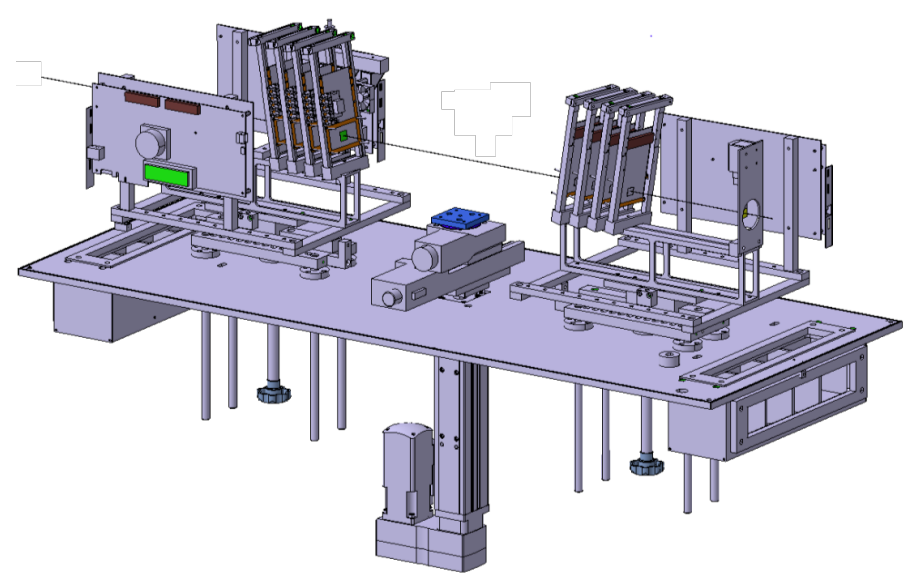

Figure 1: Drawing of the Timepix 3 telescope. A right handed co-ordinate system is used, with the $z$ axis parallel to the beam axis pointing downstream, and $y$ axis pointing upwards. to Timepix3 ASICs. This paper describes the telescope itself and some performance characteristics.

\section{The Timepix3 Telescope}

\subsection{Timepix3 Hybrid}

Each telescope plane is composed of $300 \mu \mathrm{m}$ thick p-on-n silicon sensors that are bumpbonded to Timepix 3 ASICs. The pixel pitch is $55 \mu \mathrm{m}$ and the matrix size is $256 \times 256$. The SPIDR readout system is used to acquire data from the Timepix 3 modules [2]. For use in the telescope, the acquisition mode is set such that the a Time-over-Threshold (ToT) and Time-of-Arrival (ToA) is measured for each hit pixel simultaneously. Each hit is also timestamped with a precision of 1.56 ns. Data driven readout is used for high rate tracking, with a maximum rate of 80 Mhits/s (using one SPIDR board per Timepix3 module). 

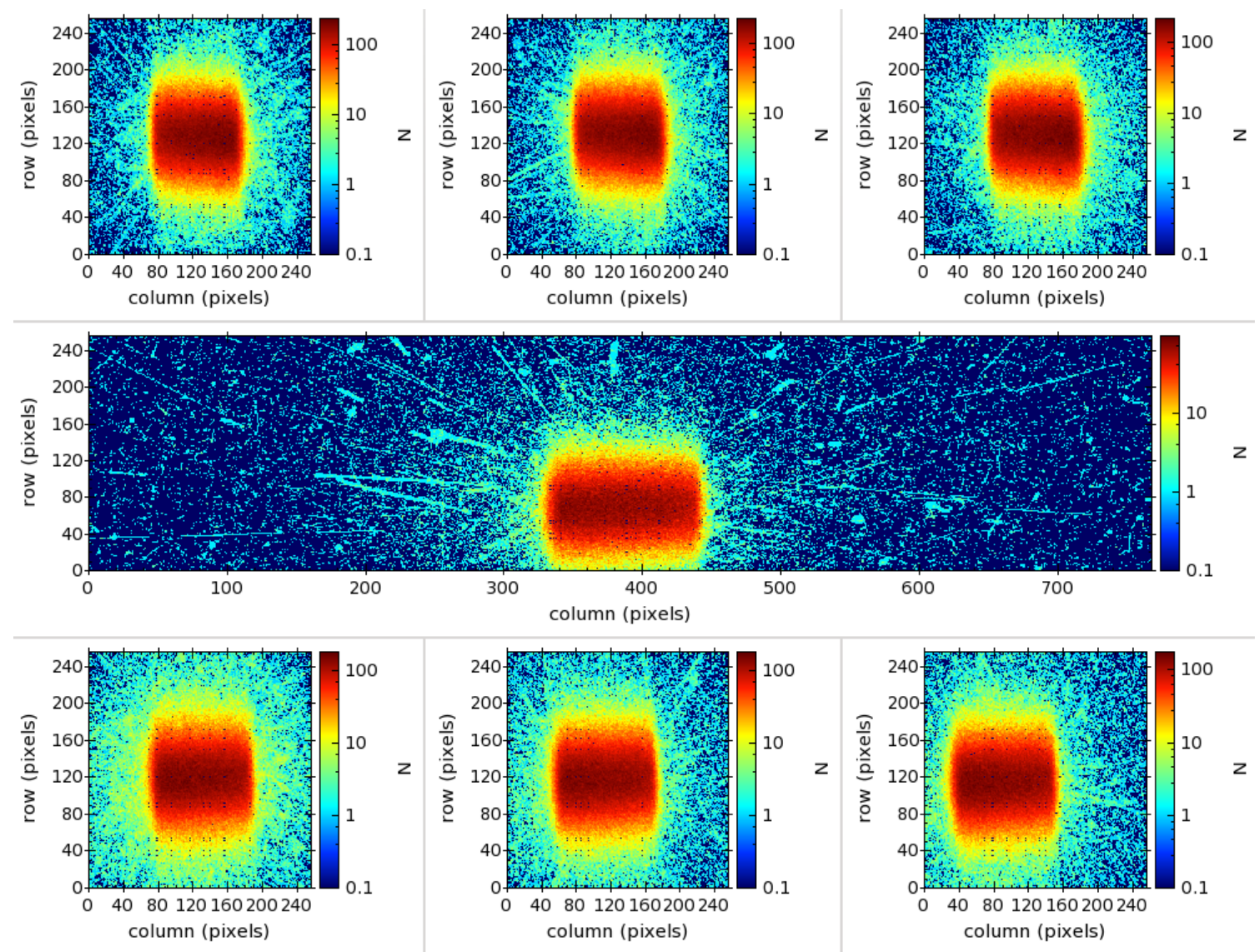

Figure 2: Screenshot of the online monitoring software used at testbeams. In this case, the device under test in the telescope was an upgrade VELO sensor bumpbonded to three Timepix3 ASICs.

\subsection{Mechanical Description}

A drawing of the Timepix 3 telescope is shown in figure 1. For optimum position resolution, the modules are tilted approximately 9 degrees in both directions perpendicular to the beam (i.e the $x$ and $y$ axes). The separation between the modules is variable, and typically set such that the distance between consecutive planes is $\sim 35 \mathrm{~mm}$. Two scintillators are also used during data taking, both upstream and downstream, providing triggers for external systems. Motion stages are placed in the middle and downstream of the telescope, allowing for translations and rotations of devices under test.

\subsection{Online Monitoring}

A small dedicated $\mathrm{C}++$ software framework has been developed for online data quality monitoring (DQM) of the telescope using Qt and ROOT libraries. Using simple and robust algorithms, the DQM ensures data taken is of good quality, via multiple measures, including:

- Ensuring the telescope and DUT is aligned with the beam using hitmaps (see figure 2).

- Checking synchronisation between telescope planes by searching for spatial and temporal correlations between hits from telescope modules. 


\subsection{Offline Reconstruction and Resolutions}

The tracking algorithm used is similar to that planned for the upgraded VELO. Track seeds are formed of pairs of non-associated clusters found from two consecutive telescope planes that are time co-incident within $10 \mathrm{~ns}$. The track seed is then extrapolated to other telescope planes to search for the remaining clusters forming the track. This reconstruction is performed in a Gaudi based [3] application has been developed to reconstruct telescope data called 'Kepler'. It has been found that the spatial resolution of a single telescope plane is around $5 \mu \mathrm{m}$, and the pointing resolution (i.e the error on the extrapolated position of the track in the centre of the telescope) is around $2 \mu \mathrm{m}$.

\subsection{Tracking Efficiency Performance}

The fraction of associated clusters is used to study the telescope tracking efficiency, since there is no accurate measure of the absolute number of tracks that pass through the telescope, and this is shown in figure 3. This has been drawn as a function of an occupancy cut, defined as the number of clusters in time co-incidence with the track. The falling trend observed is due to secondary tracks that are caused by interactions of tracks with the telescope material. These secondaries are typically angled, often falling outside of the geometrical acceptance of the telescope, resulting in additional non-associated clusters. Therefore, the fraction of associated clusters is reduced due to these clusters from secondary tracks. The fraction of associated clusters as a function of this selection is shown in figure 3. It can be seen that when demanding only eight clusters in coincidence, the fraction of associated clusters is $98 \%$, although this comes at the expense of vetoing $14 \%$ of tracks. This parameter is tuned depending on the particular study.

\section{References}

[1] The $\mathrm{LHCb}$

Collaboration, The $\mathrm{LHCb}$

Detector at the LHC, JINST 3 (2008) S08005.

[2] J. Visser

et al., SPIDR: a read-out system for Medipix3

Timepix3, JINST

10 (2015), no.12 C12028.

[3] M.

Clemencic et al., Recent

developements in the

$\mathrm{LHCb}$ software framework

Gaudi, J. Phys. Conf.

Ser. 219 (2010) 042006.

[4] The LHCb Collaboration, LHCb VELO

Upgrade Technical Design

Report, CERN, 2013.

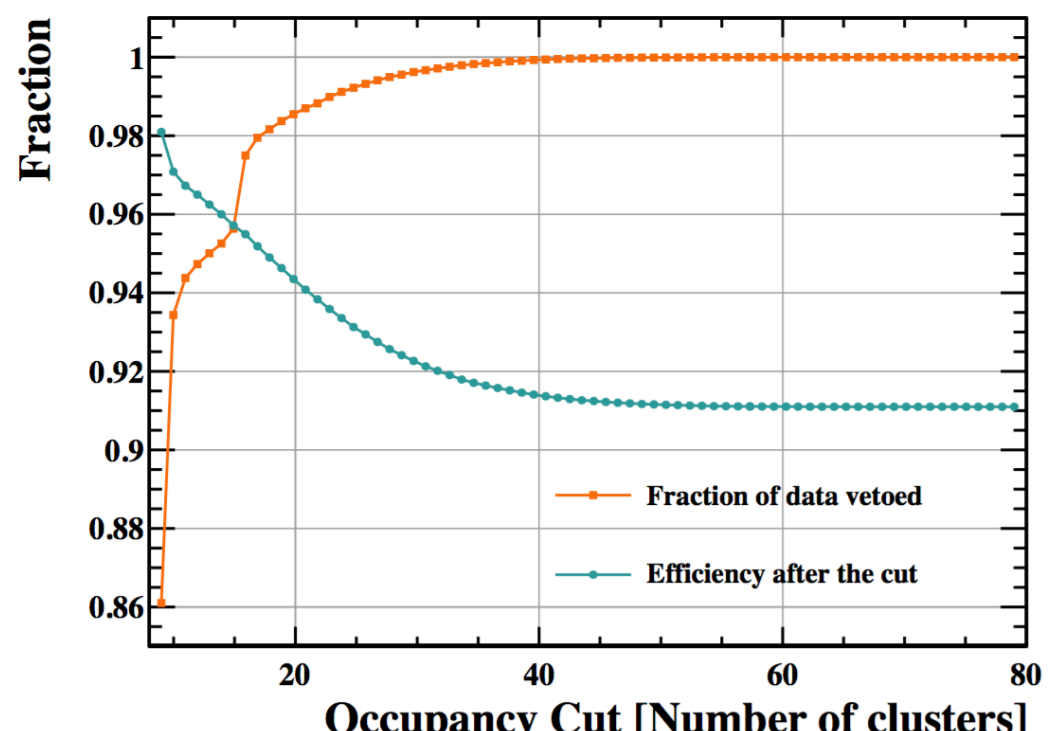

Figure 3: Fraction of associated clusters as a function of occupnacy cut. 